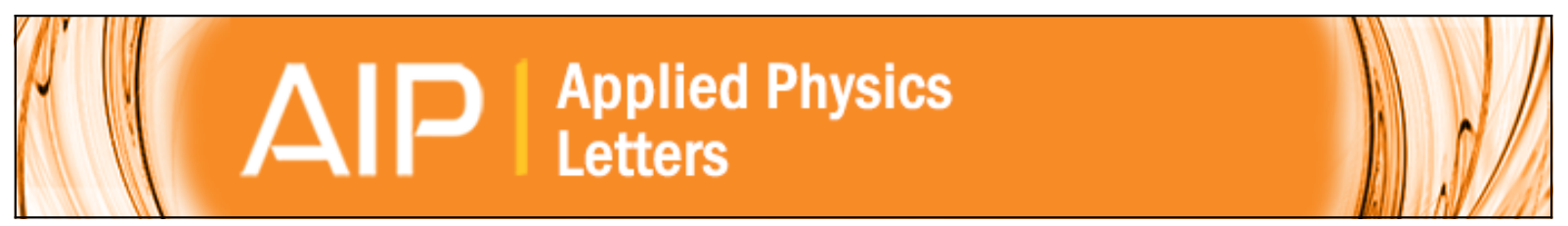

\title{
Self-limited underdense microplasmas in bulk silicon induced by ultrashort laser pulses
}

Alexandros Mouskeftaras, Andrei V. Rode, Raphaël Clady, Marc Sentis, Olivier Utéza, and David Grojo

Citation: Applied Physics Letters 105, 191103 (2014); doi: 10.1063/1.4901528

View online: http://dx.doi.org/10.1063/1.4901528

View Table of Contents: http://scitation.aip.org/content/aip/journal/apl/105/19?ver=pdfcov

Published by the AIP Publishing

\section{Articles you may be interested in}

Ultrafast dynamics of the dielectric functions of $\mathrm{ZnO}$ and $\mathrm{BaTiO} 3$ thin films after intense femtosecond laser excitation

J. Appl. Phys. 115, 053508 (2014); 10.1063/1.4864017

Temperature dependent ablation threshold in silicon using ultrashort laser pulses

J. Appl. Phys. 112, 103514 (2012); 10.1063/1.4766380

Self-limited ionization of GaAs at high femtosecond laser intensities

AIP Conf. Proc. 1464, 146 (2012); 10.1063/1.4739869

Thermoplastic deformation of silicon surfaces induced by ultrashort pulsed lasers in submelting conditions J. Appl. Phys. 111, 053502 (2012); 10.1063/1.3688020

Terahertz radiation from a magnetoplasma wave excited by ultrashort optical pulses

J. Appl. Phys. 107, 113505 (2010); 10.1063/1.3393998

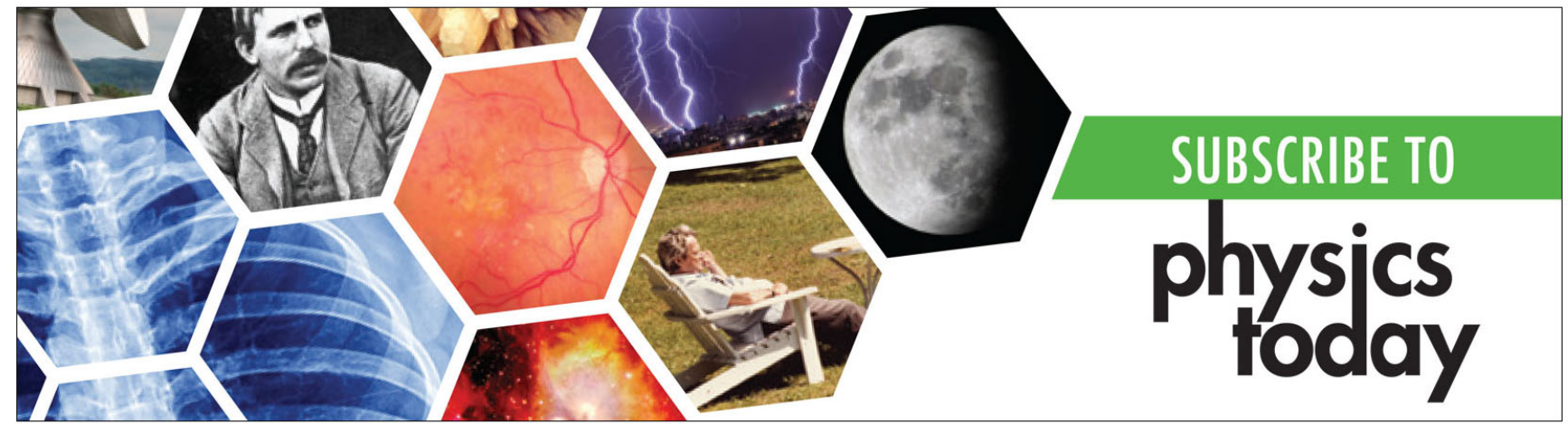




\title{
Self-limited underdense microplasmas in bulk silicon induced by ultrashort laser pulses
}

\author{
Alexandros Mouskeftaras, ${ }^{1}$ Andrei V. Rode,${ }^{2}$ Raphaël Clady, ${ }^{1}$ Marc Sentis, ${ }^{1}$ Olivier Utéza, ${ }^{1}$ \\ and David Grojo ${ }^{1, a)}$ \\ ${ }^{1}$ CNRS, LP3 UMR 7341, Aix-Marseille University, F-13288 Marseille, France \\ ${ }^{2}$ Laser Physics Centre, Research School of Physics and Engineering, Australian National University, \\ Canberra ACT 0200, Australia
}

(Received 4 September 2014; accepted 31 October 2014; published online 10 November 2014)

\begin{abstract}
Two-photon ionization by focused femtosecond laser pulses initiates the development of micrometer-scale plasmas in the bulk of silicon. Using pump-and-probe transmission microscopy with infrared light, we investigate the space-time characteristics of these plasmas for laser intensities up to $10^{12} \mathrm{~W} / \mathrm{cm}^{2}$. The measurements reveal a self-limitation of the excitation at a maximum free-carrier density of $\cong 10^{19} \mathrm{~cm}^{-3}$, which is more than one order of magnitude below the threshold for permanent modification. The plasmas remain unchanged in the $\sim 100 \mathrm{ps}$ timescale revealing slow carrier kinetics. The results underline the limits in local control of silicon dielectric permittivity, which are inherent to the use of single near-infrared ultrashort Gaussian pulses. C) 2014 AIP Publishing LLC. [http://dx.doi.org/10.1063/1.4901528]
\end{abstract}

In the past two decades, high ${ }^{1}$ (up to $\sim \mathrm{MJ} / \mathrm{cm}^{3}$ ) and localised $^{2}$ (down to $0.008 \mu \mathrm{m}^{3}$ volumes) energy concentrations inside non-conducting solids have been demonstrated. At these energy densities, sub-bandgap wavelength light can be used to locally generate free carriers by nonlinear interaction, thus transforming an otherwise transparent solid in an absorbing one. At this stage, the dielectric permittivity of the medium is changed, leading to an efficient transfer of energy from the laser to the lattice. Finally, if the deposited energy concentration exceeds the threshold for permanent modification, irreversible damage may take place. As a result, numerous applications appeared in dielectrics including optical storage $^{2}$ and the direct writing of waveguides ${ }^{4,5}$ and other integrated optical functionalities. ${ }^{3}$

Silicon ( $\mathrm{Si}$ ) becoming an increasingly dominant material in the field of hybrid devices (optical and electronic), similar developments (e.g., waveguide writing) are highly desired for semiconductors. However, the development remains far behind and there is so far no demonstration of true $3 \mathrm{D}$ control of induced modifications inside Si. On the contrary, recent works report on a strong delocalization of intense light making impossible to concentrate laser energy to a level which produces irreversible changes in bulk of $\mathrm{Si}^{6}$ Nevertheless, even below the permanent modification level, reversible changes, which are the result of dynamic control of the dielectric permittivity, are beneficial for a certain number of applications including electrical circuit defect analysis ${ }^{7}$ and cryptography. ${ }^{8}$ All these processes underline the importance of quantitative information related to the transient evolution of the Si dielectric permittivity.

In this paper, we propose the study of the dielectric permittivity in bulk crystalline $\mathrm{Si}$ (c-Si) excited by near infrared (NIR) light. Ultrashort laser pulses at $1.3-\mu \mathrm{m}$ wavelength $(0.95 \mathrm{eV})$ have been focused inside the $1.1-\mathrm{eV}$ bandgap Si. The nonlinear nature of the induced ionization ${ }^{9}$ allows for

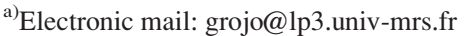

the generation of well localised carriers, at which we will be referring as microplasma, inside the crystal without observing any permanent modification. Pump-and-probe infrared imaging technique is used to study the spatio-temporal evolution of the plasmas. At this stage, let us mention that the necessary temperature for the bandgap close-up (down to $0.95 \mathrm{eV}$ ) with temperature is $\sim 850 \mathrm{~K}$ (Ref. 10) and that we are far from reaching these temperatures in our conditions as we will see later. Furthermore, our samples have been tested for defects and no significant linear absorption has been measured at $1.3-\mu \mathrm{m}$ wavelength.

As shown in Fig. 1, a Ti-Sa laser system (ASUR facility, Amplitude technologies) is delivering $30 \mathrm{fs}$ pulses at $800 \mathrm{~nm}$

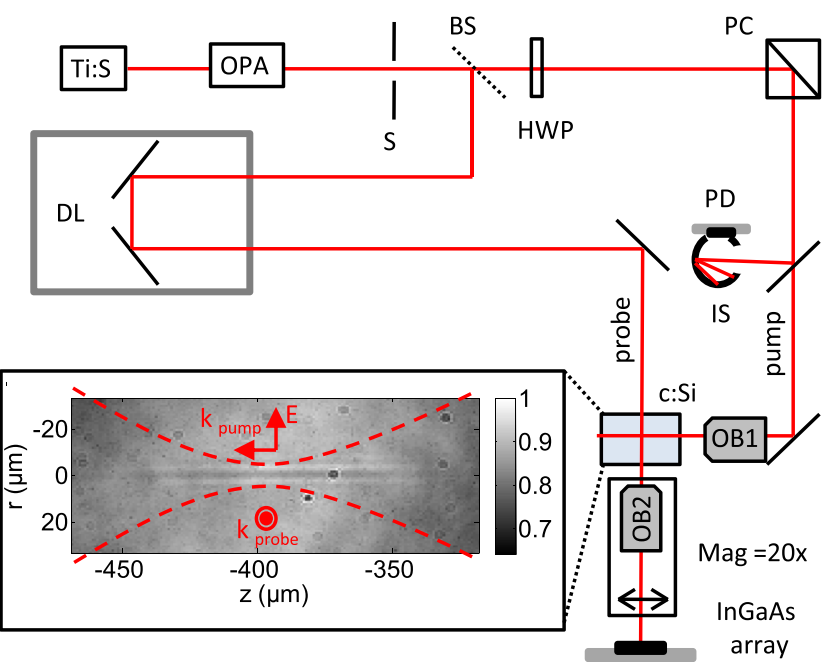

FIG. 1. Experimental setup using: (Ti:S) Ti:sapphire femtosecond laser; (S) mechanical shutter electrically commanded; (OPA) optical parametric amplifier for wavelength conversion at $1.3 \mu \mathrm{m}$; (BS) 10:90 beamsplitter; (HWP) half waveplate; (DL) delay line; (PD) photodiode; (OB) objective; (IS) integrating sphere; (PC) polarizing cube; and (ES) energy sensor. Inset: Intensity of the transmitted probe (a.u.) in presence of a pump pulse (10 nJ, 10 ps delay) as acquired with the sensor. The microplasma is visible (black stripe) at the focus of the pump beam. 
with a repetition rate of $100 \mathrm{~Hz}$. A high energy optical parametric amplifier (OPA) system (TOPAS-HE, Light Conversion) is used to tune the wavelength to $1.3 \mu \mathrm{m}$ yielding linearly polarised pulses with energy up to $1 \mathrm{~mJ}$ and duration $\tau \cong 80 \mathrm{fs}$ (FWHM) as measured using a single shot auto-correlator (TiPAAT5C3, Light Conversion). A beamsplitter reflects $10 \%$ of the beam to create the probe pulse. The remaining part (pump) propagates along a half-wave plate and a polarising cube combination to control the incident energy, measured with an integrating sphere and a photodiode. Our measurements are reported in terms of energy delivered at the focus as if no sample were in place (reflection from the sample is not being taken into account).

The pump pulse is focused (NA=0.3) with a NIR microscope objective (Olympus, LMPLN10XIR). The sample is an intrinsic c-Si wafer (Siltronix) with $200 \Omega \cdot \mathrm{cm}$ resistivity, (100) orientation, and $1 \mathrm{~mm}$ thickness. The transmitted pump intensity is measured with a highsensitivity photodiode energy sensor (OPHIR, PD10-IR-PJ). Then, using a so-called Z-scan transmission measurement procedure, we can position precisely the pump beam focus at a depth of $400 \mu \mathrm{m}$ below the surface of the sample before the experiments. ${ }^{11}$ This ensures sufficiently low intensity on the surface to investigate only bulk effects. Motorised stages ensure sample translation between shots so that each single pulse interacts with a fresh material. Before the plasma investigations, we used a high resolution imaging setup (not shown) to fully characterize the laser intensity distribution in the focal region. We found a nearly Gaussian distribution with spot size and confocal parameter of $4 \mu \mathrm{m}$ and $20 \mu \mathrm{m}$ (FWHM) in air respectively (corresponding to $4 \mu \mathrm{m}$ and $70 \mu \mathrm{m}$ in $\mathrm{Si}$ ). Finally, lateral imaging of the interaction region is made with a $20 \times$ magnification (LMPLN20XIR microscope objective, Olympus) on an InGaAs detector (XenicsXeva) yielding observations with $\cong 1.4 \mu \mathrm{m}$ spatial resolution.

According to the linear response of our detector, we display directly the probe transmission through the plasma by normalisation of the images using the signal outside the plasma (background signal). The plasma transmission retrieval assumes that reflection from the generated plasma is negligible, which is reasonable for $N<10^{20} \mathrm{~cm}^{-3}$ yielding $R<1 \%$. To avoid any false considerations, we have also performed calibration measurements (not shown here) to verify the linearity of probe absorption by the plasma at the intensity used for the imaging experiments. Then, acquiring images for different pump-probe delays and pump energies allows us to investigate the microplasmas.

A time-resolved transmission measurement along the optical axis of the pump (longitudinal direction of the plasma) is shown in Fig. 2. One can see a significant drop over the first $\sim 130 \mathrm{ps}$. Since thermal equilibrium between free-carriers and the lattice can be rather fast in $\mathrm{Si}^{12}$ $(\sim 500 \mathrm{fs})$, the first step was to estimate the influence of a potential bandgap close-up due to pump energy transfer in the focal volume. The difference between pump photon and bandgap energy being $\sim 150 \mathrm{meV}$, linear absorption could arise from a temperature rise of $\cong 550 \mathrm{~K}$. With simple thermodynamic considerations, one can estimate the maximum local heating in the experiments as $\Delta T\left({ }^{\circ} \mathrm{C}\right)=E /(V \times \rho \times$

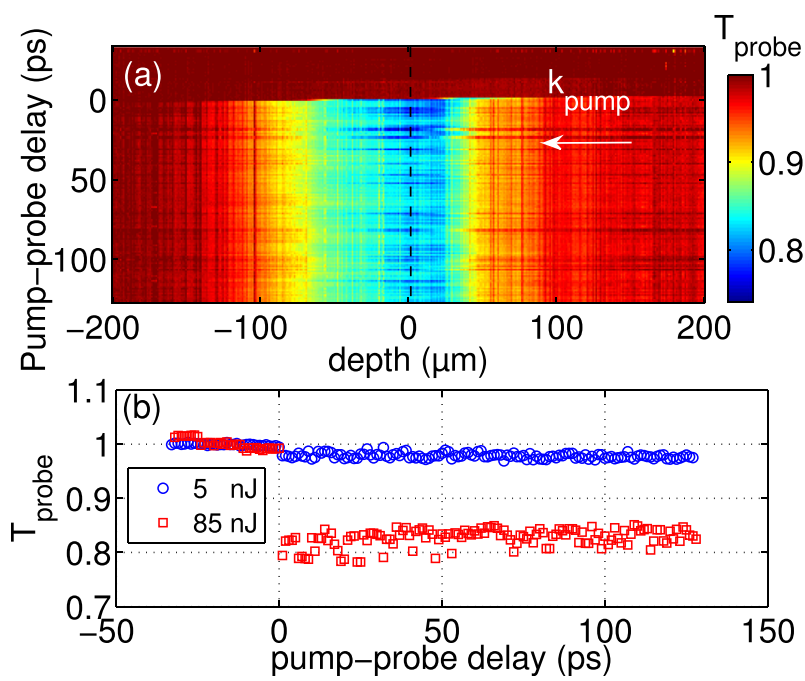

FIG. 2. (a) Probe transmission along the optical axis of the pump (depth) versus pump-probe delay inside Si. Depth axis is translated so that zero corresponds to $400 \mu \mathrm{m}$ below the surface. Pump energy is $85 \mathrm{~nJ}$. (b) The transmission measured at the center of the micro-plasma is plotted (dotted line on Fig. 2(a)), as a function of delay, for two different pump pulse energies: $5 \mathrm{~nJ}$ and $85 \mathrm{~nJ}$.

$C)$ with $E$ as the total pump energy, $V$ as the interaction volume (modelled by a cylinder $100 \mu \mathrm{m}$ long over a $3 \mu \mathrm{m}$ diameter, see inset of Fig. 1), $\rho$ as the Si density $\left(2.33 \mathrm{~g} \cdot \mathrm{cm}^{-3}\right)$, and $C$ as the thermal capacity $\left(0.712 \mathrm{~J} \cdot \mathrm{g}^{-1} \cdot \mathrm{C}^{-1}\right)$. This yields a potential local heating $\leq 1 \mathrm{~K}$ in the framework of our experiments (pump energy $\leq 100 \mathrm{~nJ}$ ) and thus we can neglect any thermal contribution to the observed absorption.

Free from this consideration, we can now attribute the observed probe absorption to inverse Bremsstrahlung absorption by the free-carriers generated by two-photon absorption (TPA) of the pump pulse. ${ }^{13}$ According to the time-resolved response at the theoretical focus shown in Fig. 3 , we measure a rise time of the absorption of $\cong 300 \mathrm{fs}$. This compares favourably with the convolution product of the pump and probe pulses that are stretched before to reach the interaction region due to dispersion in Si. For comparison, we have measured that our pulses are stretched up to $120 \mathrm{fs}$

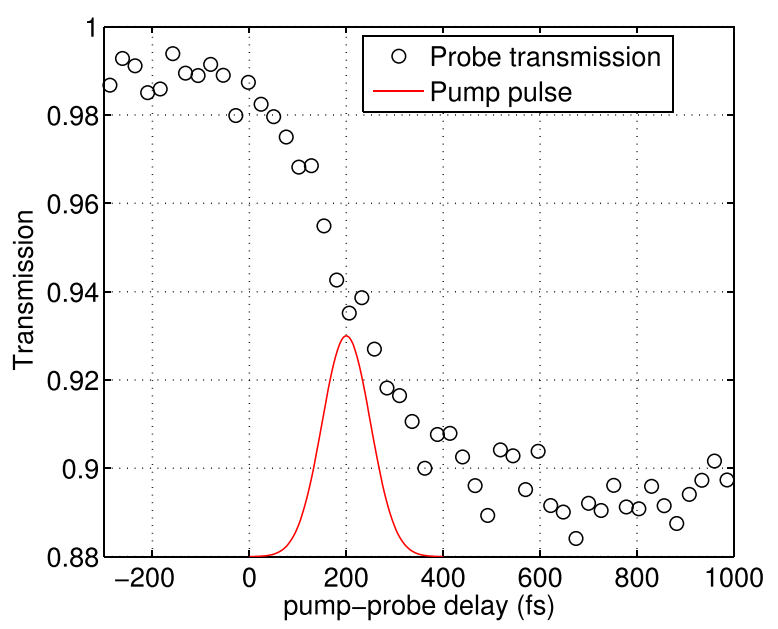

FIG. 3. Probe transmission along the center of the micro-plasma as a function of the pump and probe delay. Pump pulse energy is $\sim 18 \mathrm{~nJ}$. For comparison, the solid line displays the temporal intensity profile of a typical $80 \mathrm{fs}$ pulse (FWHM). 
after propagation inside a $1 \mathrm{~mm}$ thick $\mathrm{Si}$ plate. Fig. 2(b) shows the time-evolution of transmission at longer pumpprobe delays for low $(5 \mathrm{~nJ})$ and high $(85 \mathrm{~nJ})$ pump pulse energies. From these data, we estimate that the stability of our measurement is $\pm 3 \%$ of the signal but no transient evolution of the absorption is observed within this time scale. Among the processes that can lead to a decrease of the excitation density and by extension a decay in absorption, carrier diffusion is believed to play an important role due to the high electron diffusion coefficient $D_{\mathrm{e}}$ of semiconductors. Using a mobility $\mu_{e}=1400 \mathrm{~cm}^{2} / \mathrm{V} \cdot \mathrm{s}$ associated to intrinsic $\mathrm{Si}$ at room temperature, ${ }^{14}$ the Einstein relation gives $D_{\mathrm{e}} \cong 36 \mathrm{~cm}^{2} / \mathrm{s}$. At the maximum probe delay $t$ of $130 \mathrm{ps}$, limited by the length of the delay line used, we can predict that free electrons will have diffused over a typical distance $L=\sqrt{D_{e} \times t}$ exceeding $0.7 \mu \mathrm{m}$ that is comparable to the radial dimension of the plasmas ( $3 \mu \mathrm{m}$, see inset in Fig. 1). However, our measurements do not exhibit any significant decay showing that a simple free-carrier diffusion description does not hold for these microscale plasmas.

In Fig. 4(a), we show the dependence of the probe transmission on the pump energy. For the measurement, the pump-probe delay is fixed at $10 \mathrm{ps}$ so that we ensure the plasma is fully established and we measure the transient absorption level that decays at much longer time scale (see Fig. 2(b)). Raising the energy, we see that the absorption front is shifted towards the prefocal region (see arrow to guide the eyes). As a result, the interaction volume increases while the minimum transmission rapidly drops down to a saturation level of $T \cong 80 \%$. This may be better appreciated on Fig. 4(b) where the plasma transmission at the theoretical focus of the pump is plotted as a function of the pump energy. For energies exceeding $30 \mathrm{~nJ}$, the transmission remains constant within the range of stability of our measurements.

The measured transmission level in the time-resolved images allows us to estimate the generated free-carrier
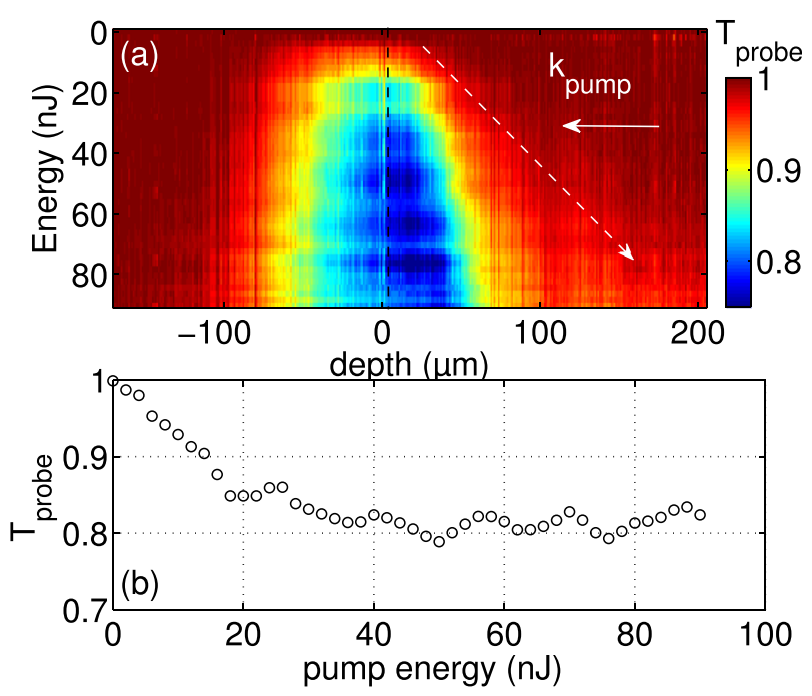

FIG. 4. (a) Probe transmission as a function of the pump pulse energy and the depth inside Si. Pump-probe delay is fixed at $10 \mathrm{ps}$. Dashed arrow follows the shift of the absorption front. Zero depth corresponds to $400 \mu \mathrm{m}$ below the Si surface. (b) The transmission at the center of the micro-plasma is plotted as a function of the pump pulse energy (dotted line on Fig. 4(a)). density $N$ in the solid. According to the Drude model, ${ }^{15}$ the complex refractive index $\tilde{n}$ can be expressed as $\tilde{n}^{2}=n_{0}^{2}-\frac{\omega_{\mathrm{p}}^{2}}{\omega^{2}+i \omega \gamma}$, with plasma frequency $\omega_{\mathrm{p}}=\sqrt{\frac{N e^{2}}{\varepsilon_{0} m^{*}}}$ where $\omega$ is the laser frequency, $e$ is the electron charge, $\varepsilon_{0}$ is the vacuum permittivity, $m^{*}$ is the effective electron mass $\left(m^{*}=0.18 m_{e}\right.$ for low-density plasma in $\left.\mathrm{Si}\right),{ }^{16}$ and $\gamma$ is the collision frequency. We choose $\gamma=0.3 \mathrm{fs}^{-1}$ corresponding to a rate dominated by electron-hole collisions, which is reasonable for intermediate to low excitation densities. A sensitivity analysis to this parameter gives less than $50 \%$ variation in the density estimation using $\gamma$ in the range $0.12-0.5 \mathrm{fs}^{-1}$ corresponding to its upper and lower limits. ${ }^{17}$ Then, assuming a homogeneous plasma, the transmission $T$ is described by a simple Beer-Lambert law, $T=\exp \left(-\frac{4 \pi \operatorname{Im}(\tilde{n})}{\lambda} d_{\text {plasma }}\right)$ where $\operatorname{Im}($.) denotes the imaginary part, $\lambda$ is the probe wavelength $(1.3 \mu \mathrm{m})$ and $d_{\text {plasma }}$ is the measured plasma diameter $(3 \mu \mathrm{m})$. Under these assumptions, the $80 \%$ minimum transmission $T_{\text {min }}$ measured (Fig. 4(b)) corresponds to an increase of the imaginary part of the refractive index from $<10^{-6}$ to $7.7 \times 10^{-3}$ at $1.3 \mu \mathrm{m}$ and a free-carrier density $N_{\max }$ of $3.1 \times 10^{19} \mathrm{~cm}^{-3}$. This reveals a limitation of the excitation to an underdense plasma with density one order of magnitude below the critical density $N_{\text {crit }}$ at $1.3 \mu \mathrm{m}\left(6.6 \times 10^{20} \mathrm{~cm}^{-3}\right)$ consistent with the impossibility to induce local breakdown inside Si with single ultrashort pulses. ${ }^{6}$

One obvious contribution to this limitation lies in beam energy depletion by TPA before the focus. The sensitivity of our diagnostic allows us to observe that the plasma develops in a pre-focal region for pump pulse energies from $\sim 40 \mathrm{~nJ}$ (see arrow in Fig. 4(a)). In addition, it is worth noting that the pump pulse energy has been in most of the measurements above the threshold for self-focusing. According to the nonlinear refractive index in $\mathrm{Si}^{13}\left(n_{\mathrm{NL}}=3 \times 10^{-14} \mathrm{~cm}^{2} / \mathrm{W}\right)$, the critical power for self-focusing ${ }^{18}$ is only $24 \mathrm{~kW}$ corresponding to a pump energy $E_{\mathrm{TH}} \cong 1 \mathrm{~nJ}$ for $80 \mathrm{fs}$ pulses. However, we did not observe any significant focus shift or deviation from Gaussian focusing in the images. Moreover, the spectrum of the transmitted pump did not exhibit any significant change. Taken together, these observations indicate that nonlinear propagation effects remain limited and the limitation of excitation is likely associated to intensity clamping by TPA.

To connect the plasma characteristics to the absence of permanent material modification, one can turn to energy considerations. Assuming that each free electron is generated by consumption of two pump photons, the absorbed energy density can be expressed as $U=2 \hbar \omega N$. If we consider the above reported $N_{\max }$, we find $U_{\max } \cong 9 \mathrm{~J} / \mathrm{cm}^{3}$, which is three orders of magnitude smaller than the typical threshold density for Si melting ${ }^{19}\left(U_{\text {fus }}=5 \mathrm{~kJ} / \mathrm{cm}^{3}\right)$.

Then, a way to further reveal that this low energy density relies on inefficient beam delivery at the focus is to compare this result to the pump beam absorption for the same experimental conditions. Figure 5 shows normalized (to the low signal) pump transmission $T_{\text {pump }}$ measurements as a function of incoming pulse energy. This allows retrieving the total deposited energy inside the solid $E_{\text {dep,tot }}=$ $E_{\text {pump }} \times\left(1-T_{\text {pump }}\right)$ as it is shown on the same graph. For 


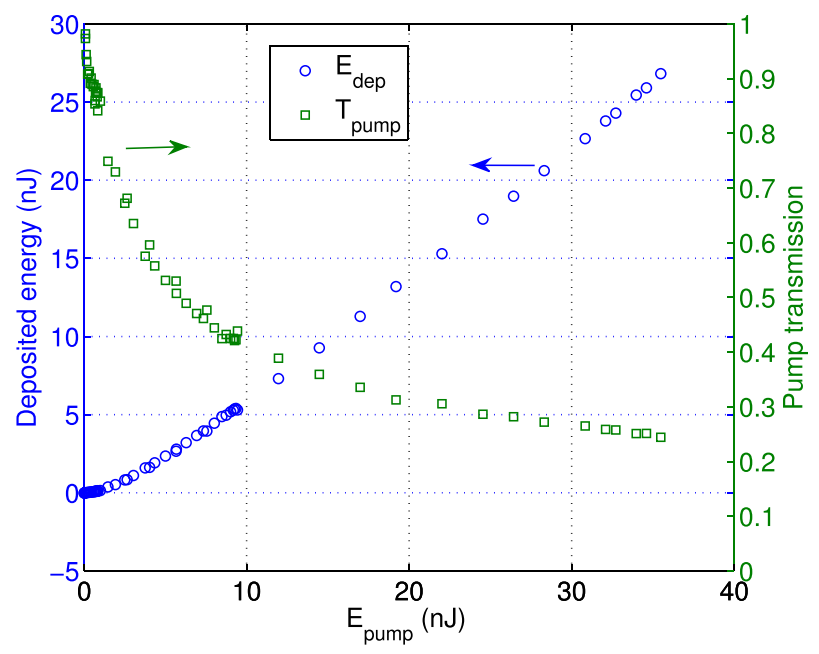

FIG. 5. Normalized transmission of the pump pulse as a function of pulse energy (right axis) measured for the same experimental conditions as for the imaging experiments. The corresponding total deposited energy is plotted on the same graph (left axis).

comparison, the maximum deposited energy to create the plasma observed in the experiments is directly given by $E_{\text {dep,focus }}=U_{\max } \times V$ where $V$ is the cylindrical volume in which the plasma is confined as determined directly from the images. Then, the ratio $\eta=E_{\text {dep,focus }} / E_{\text {dep,tot }}$ stands for the efficiency of the microplasma formation. For two different incoming pump energies corresponding to a low excitation $(20 \mathrm{~nJ})$ and the saturation regime $(36 \mathrm{~nJ})$, this leads to a ratio of respectively: $27 \%$ and $25 \%$ only, which underlines that most of the energy losses from the pump do not contribute to the formation of the plasma observed in the experiments. This is likely another indication of the pre-focal pump beam absorption as above highlighted.

In conclusion, space-time control of the dielectric permittivity is the first step towards 3D material processing in c-Si. Our experiments show local modification of the imaginary part of the refractive index. However, we observe considerably low absorption levels independently of the incoming pulse energy. Moreover, our time-resolved study of this absorption reveals very slow carrier kinetics and no significant recombination within the 130 ps after the laser excitation. This reveals strong limitations when considering bulk excitation of $\mathrm{Si}$ by the use of single NIR ultrafast Gaussian pulses. While we have concentrated on measuring the inherent characteristics of the microplasmas, we will focus a future paper on conditions for improved energy confinement. Next, natural step will be to explore how our observations translate using tighter focusing conditions. We envisage also that the formation of microplasmas, similar to that in the inset in Fig. 1, can serve as a strongly absorbing media for efficient energy deposition in $\mathrm{Si}$ with a second synchronized pulse as it is proposed for ultrafast dielectric interactions. $^{20}$

This research has received financial support from the French National Research Agency (ANR 2010-JCJC-91301), the French Carnot Star Institute (Via-LASER), and the CNRS PICS Program No. 45052. A.R. is grateful for partial support by the Australian Research Council's (Discovery Project DP 120102980) and by the Air Force Office of Scientific Research (USA, Grant No. FA 9550-12-1-0482).

${ }^{1}$ E. G. Gamaly, S. Juodkazis, K. Nishimura, H. Misawa, B. Luther-Davies, L. Hallo, P. Nicolai, and V. T. Tikhonchuk, Phys. Rev. B 73, 214101 (2006).

${ }^{2}$ E. N. Glezer, M. Milosavljevic, L. Huang, R. J. Finlay, T.-H. Her, J. P. Callan, and E. Mazur, Opt. Lett. 21, 2023-2025 (1996).

${ }^{3}$ R. R. Gattass and E. Mazur, Nat. Photonics 2, 219-225 (2008).

${ }^{4}$ K. M. Davis, K. Miura, N. Sugimoto, and K. Hirao, Opt. Lett. 21, 1729-1731 (1996).

${ }^{5}$ K. Miura, J. R. Qiu, H. Inouye, T. Mitsuyu, and K. Hirao, Appl. Phys. Lett. 71, 3329-3331 (1997).

${ }^{6}$ V. V. Kononenko, V. V. Konov, and E. M. Dianov, Opt. Lett. 37, 3369 (2012).

${ }^{7}$ C. Xu and W. Denk, Appl. Phys. Lett. 71, 2578-2580 (1997).

${ }^{8}$ A. P. Mirbaha, Ph.D. dissertation, Ecole nationale supérieure des mines de Saint-Etienne, Gardanne, 2011.

${ }^{9}$ D. Grojo, S. Leyder, P. Delaporte, W. Marine, M. Sentis, and O. Utéza, Phys. Rev. B 88, 195135 (2013).

${ }^{10}$ S. M. Sze and K. K. Ng, Physics of Semi-Conductors Devices (Wiley, New Jersey, 2006), p. 16.

${ }^{11}$ S. Leyder, D. Grojo, P. Delaporte, W. Marine, M. Sentis, and O. Utéza, Appl. Surf. Sci. 278, 13-18 (2013).

${ }^{12}$ H. M. Van Driel, Phys. Rev. B 35, 8166 (1987).

${ }^{13}$ A. D. Bristow, N. Rotenberg, and H. M. Van Driel, Appl. Phys. Lett. 90, 191104 (2007).

${ }^{14}$ N. D. Arora, J. R. Hauser, and D. J. Roulston, IEEE Trans. Electron. Devices 29, 292-295 (1982).

${ }^{15}$ P. Drude, Annalen der Physik 306, 566-613 (1900).

${ }^{16}$ K. Sokolowski-Tinten and D. von der Linde, Phys. Rev. B 61, 2643-2650 (2000).

${ }^{17}$ A. Rämer, O. Osmani, and B. Rethfeld, J. Appl. Phys. 116, 053508 (2014).

${ }^{18}$ J. H. Marburger, Prog. Quantum Electron. 4, 35-110 (1975).

${ }^{19}$ K. Yamaguchi and K. Itagaki, J. Therm. Anal. 69, 1059-1066 (2002).

${ }^{20}$ X. A. Shen, S. C. Jones, and P. Braunlich, Phys. Rev. Lett. 62, 2711 (1989). 